\title{
The latest results on top quark pair cross-section measurement at the LHC-ATLAS experiment
}

\author{
Katsuya YAMAUCHI* on behalf of the ATLAS collaboration \\ Nagoya University \\ E-mail: katsuyachepl.phys.naqova-u.ac.jp
}

The latest results on top quark pair production cross-section measurements in proton-proton collisions at $\sqrt{s}=7 \mathrm{TeV}$ and $\sqrt{s}=8 \mathrm{TeV}$ with the ATLAS detector at the LHC are reported. The inclusive cross-section was measured with relative uncertainty of $4 \%$ using the final state of the top quark pair including an electron and a muon. The measurement of the differential crosssection as functions of various observables such as the transverse momentum and the rapidity of the top quark and the invariant mass of the top quark pair system are also reported. These results are compared with various generators such as POWHEG, ALPGEN and MC@NLO and various sets of parton distribution functions.

Flavor Physics \& CP Violation 2015

May 25-29, 2015

Nagoya, Japan

${ }^{*}$ Speaker. 


\section{Introduction}

The top quark, whose mass is $173 \mathrm{GeV}$, is the heaviest elementary particle in the Standard Model (SM). Since its mass is close to the electro-weak symmetry breaking scale, the top quark production cross-section is sensitive to various new physics models. Additionally, since the top anti-top pairs $(t \bar{t})$ are produced via the strong interaction at Large Hadron Collider (LHC) [W], the measurement of the pair-production cross-section is useful to test the perturbative QCD (pQCD).

The top quark decays into a $b$ quark and a $W$ boson with a branching fraction of almost 100 $\%$. Therefore, the final state of the top quark pair is categorized by the decays of two $W$ bosons. All jets channel, where both $W$ bosons decay to hadronic states, has the largest statistics while poor signal-to-background ratio (S/B). Lepton+jets channel, where one $W$ boson decays to a final state with leptons and the other $W$ boson decays to hadronic state, has reasonable statistics and S/B. Dilepton channel, where both $W$ bosons decay to the final states with leptons, has the smallest statistics while the best $\mathrm{S} / \mathrm{B}$.

In this report the latest results on top quark pair cross-section measurement at the ATLAS experiment [ [ $[$ ] are reported. The analyzed data samples correspond to the integrated luminosities of $4.6 \mathrm{fb}^{-1}$ and $20.3 \mathrm{fb}^{-1}$ for $\sqrt{s}=7 \mathrm{TeV}$ and $\sqrt{s}=8 \mathrm{TeV}$, respectively.

\section{Inclusive cross-section measurements of top-pair production cross-section}

Figure $\square$ shows the summary of the inclusive measurements of the top-pair production crosssection at various center of mass energies. All the results of the measurements are consistent with the SM prediction. The most precise measurement for $\sqrt{s}=8 \mathrm{TeV}$ is based on dilepton channel, with an electron and a muon in the final state, $\sigma_{t \bar{t}}=242.4 \pm 1.7$ (stat.) \pm 5.5 (syst.) \pm 7.5 (lumi.) \pm 4.2(beamenergy) $\mathrm{pb}[$ [B]].

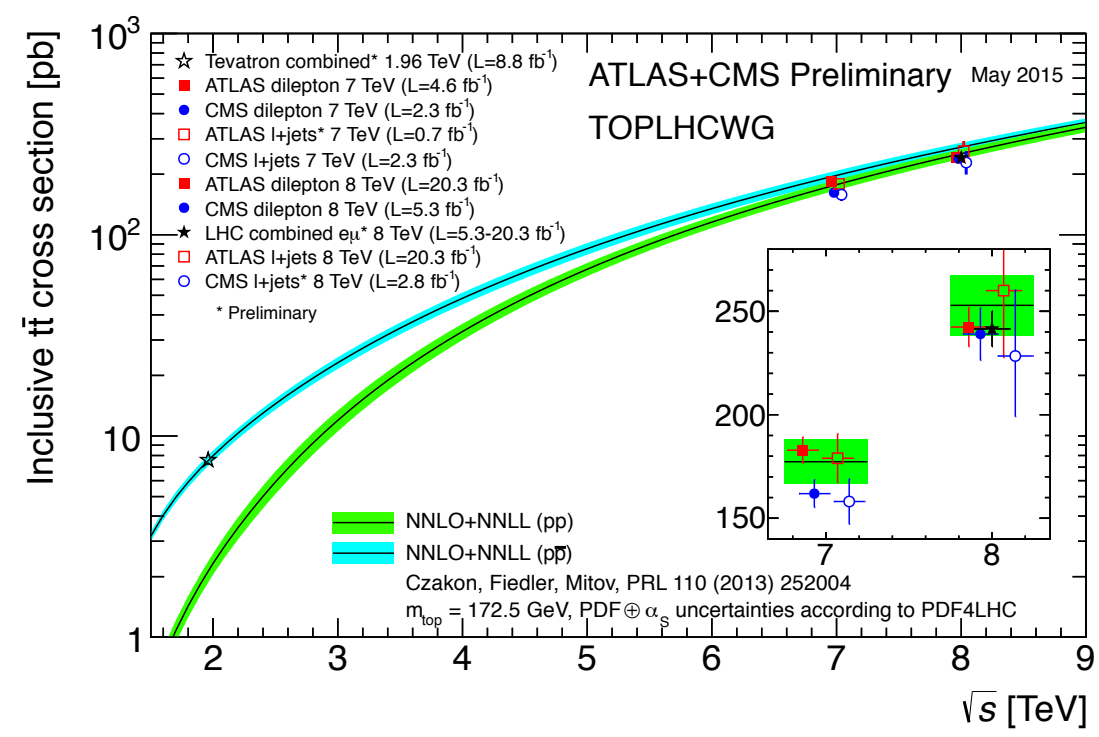

Figure 1: Summary of the inclusive measurements of the top-pair production cross-section [四]. The horizontal axis shows the center of mass energy of proton-proton collision. The mass of top quark $\left(m_{t}\right)$ is assumed to be $172.5 \mathrm{GeV}$ for these measurements and theoretical calculations. 


\section{Differential cross-section measurement using lepton+jets events at $7 \mathrm{TeV}$}

The normalized differential cross-section for top quark pair production is measured using lepton+jets events at $7 \mathrm{TeV}$ [5]. In this measurement kinematic variables of top quark pair is obtained with a likelihood fit. Top quark decay in a leading-order (LO) representation is assumed in the likelihood fit. Reconstructed variables are unfolded to correct for the detector response.

Figure $\square$ shows the measured normalized differential cross-sections as a function of the transverse momentum of the hadronically decaying top quark $\left(p_{\mathrm{T}}^{t}\right)$ and the invariant mass of the top quark pair system $\left(m_{t \bar{t}}\right)$. Various Monte Carlo (MC) generator predictions are compared with the measured cross-section. Figure 3$]$ shows the ratios of the next-to-leading order (NLO) QCD predictions to the measured normalized differential cross-sections depending on $p_{\mathrm{T}}^{t}$ and $m_{t \bar{t}}$ for different sets of parton distribution functions. MC predictions agree with the data within the uncertainties. However, the measured cross-sections are smaller than the predictions in high $p_{\mathrm{T}}^{t}$ and $m_{t \bar{t}}$ regions. The distributions show some preference for HERAPDF1.5.

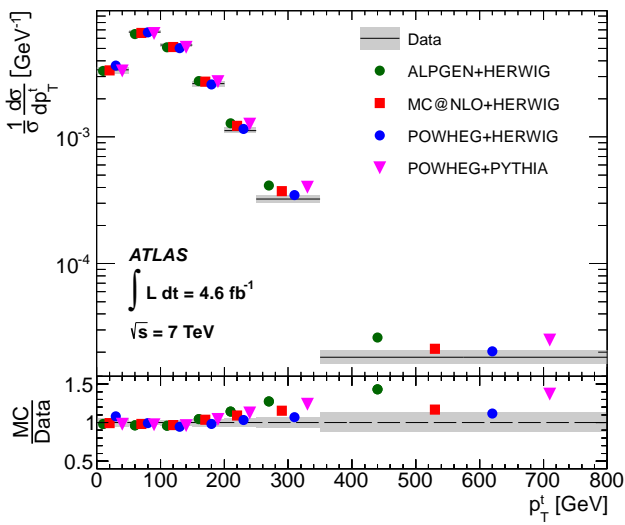

(a)

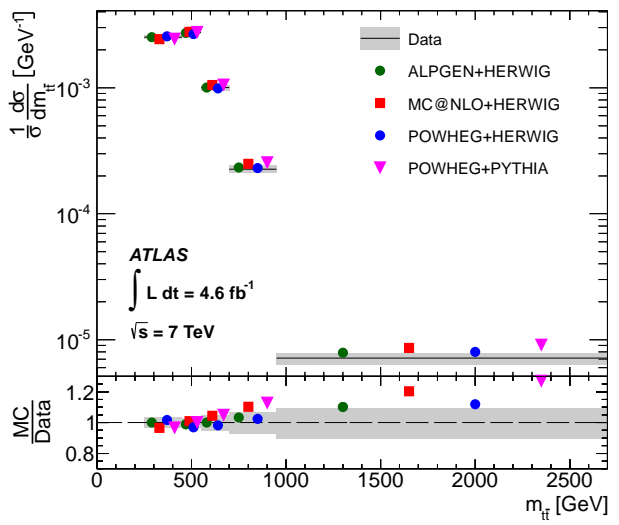

(b)

Figure 2: Normalized differential cross-sections as a function of the transverse momentum of the hadronically decaying top quark $\left(p_{\mathrm{T}}^{t}\right)(\mathrm{a})$, and the invariant mass of the top quark pair system $\left(m_{t \bar{t}}\right)$ (b) [[]]. Generator predictions are compared with ALPGEN+HERwIG [ [0, प], MC@ NLO+HERwIG [8]], POWHEG+HERWIG [Q] and Powheg+Pythia [0]].

\section{Differential cross-section measurement for boosted top quark using lepton + jets events at $8 \mathrm{TeV}$}

The differential cross-section as a function of $p_{\mathrm{T}}^{t}$ is measured for the hadronically decaying top quark with $p_{\mathrm{T}}>300 \mathrm{GeV}[\mathbb{\mathrm { L }}]$ ]. The jets from high- $p_{\mathrm{T}}$ top quarks are collimated and therefore treated as a single jet with large radius. The $p_{\mathrm{T}}$ distribution of the large-radius jet is unfolded to correct for the detector response. Both of the parton level and particle level cross-sections are measured.

Figure T.a shows the particle-level differential cross-sections as a function of the transverse momentum of the top quark $\left(p_{\mathrm{T}}^{t}\right)$. Figure 目. b shows the parton-level differential cross-sections as a function of the transverse momentum of the top quark $\left(p_{\mathrm{T}}^{t}\right)$. The measured cross-sections are 


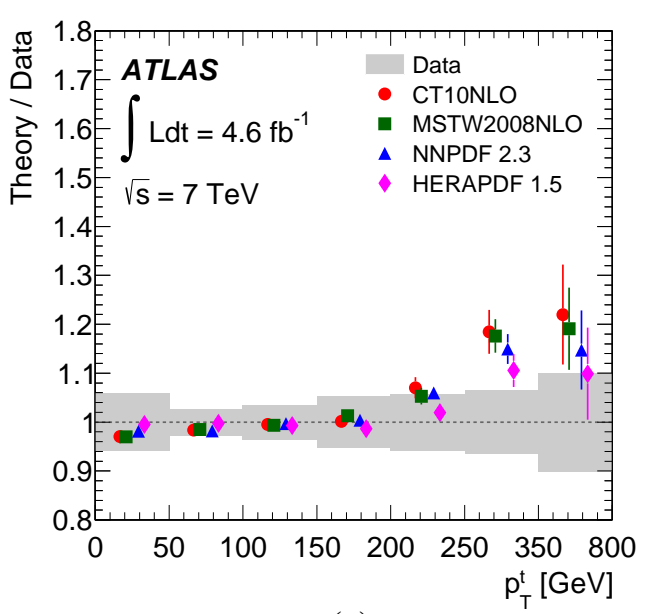

(a)

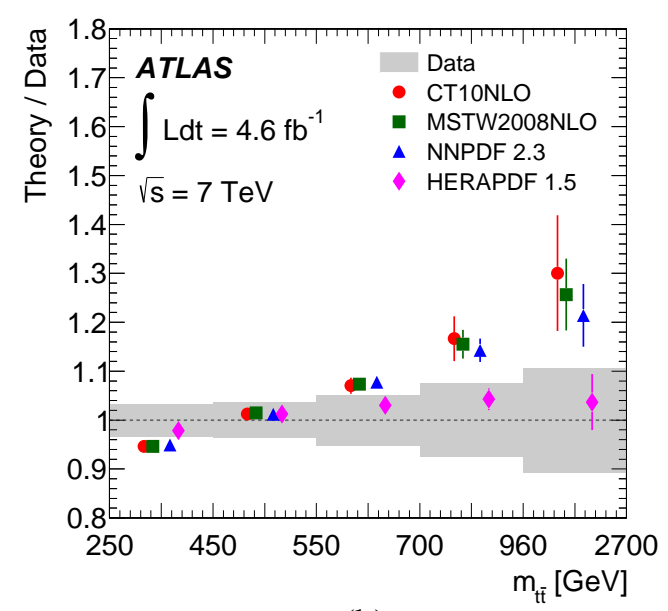

(b)

Figure 3: Ratios of the NLO QCD predictions to the measured normalized differential cross-section depending on $p_{\mathrm{T}}^{t}$ and $m_{t \bar{t}}$ for different PDF (CT10 [ய]], MSTW2008NLO [ए2], NNPDF2.3 [ए3]], HERAPDF1.5 [미]) sets [[]].

generally smaller than the prediction of NLO and LO + parton shower MC generators after normalization to next-to-next-to-leading order (NNLO) + next-to-next-to-leading logarithmic (NNLL) QCD calculations of the inclusive cross-section. The discrepancy between the measurements and the predictions ranges from $30 \%$ to $70 \%$ in the highest- $p_{\mathrm{T}}$ bin, depending on MC generators employed in the predictions.

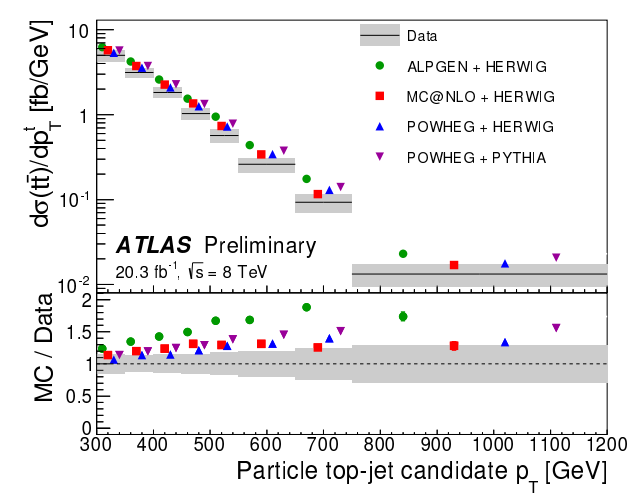

(a)

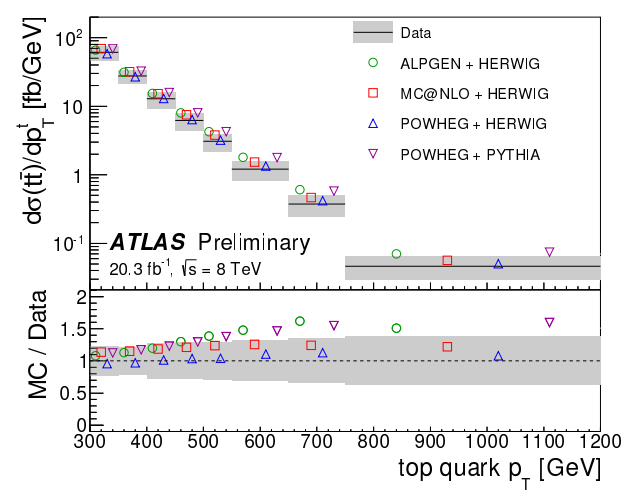

(b)

Figure 4: Particle-level differential cross-section as a function of the $p_{\mathrm{T}}^{t}$ (a). Parton-level differential crosssection as a function of the $p_{\mathrm{T}}^{t}(\mathrm{~b})$. Each measured cross-section is compared with ALPGEN+HERWIG,

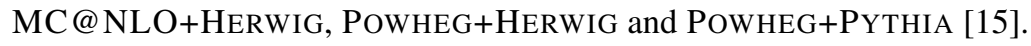

\section{Conclusion}

Large amount of top quark pairs allow us to measure the differential top quark pair production cross-section as a function of the transverse momentum of the top quark, and the invariant 
mass of the top quark pair system. The measurements are generally consistent with the SM prediction within the uncertainties. The measured cross-section tends to be smaller than the theoretical prediction for both $7 \mathrm{TeV}$ and $8 \mathrm{TeV}$ data in a high momentum region.

\section{References}

[1] L. Evans and P. Bryant, "LHC Machine”, JINST 3 S08001 (2008).

[2] ATLAS Collaboration, "The ATLAS Experiment at the CERN Large Hadron Collider", JINST 3 S08003 (2008).

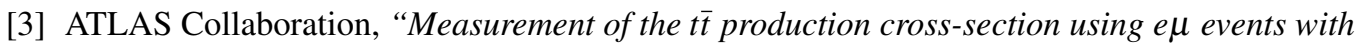
$b$-tagged jets in pp collisions at $\sqrt{s}=7$ and 8 TeV with the ATLAS detector", Eur. Phys. J. C74 (2014) 3109, [arXiv: 1406.5375$]$.

[4] TOP LHC WG Summary Plots are available at [https://twiki.cern.ch/twiki/bin/view/LHCPhysics/TopLHCWGSummaryPlots].

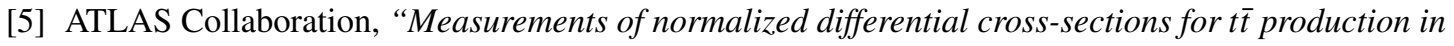
pp collisions at $\sqrt{s}=7 \mathrm{TeV}$ using the ATLAS detector", Phys. Rev. D90 (2014) 072004 [arXiv:1407.0371].

[6] M. L. Mangano et al., "ALPGEN, a generator for hard multiparton processes in hadronic collisions", JHEP 0307 (2003) 001, [arXiv: hep-ph/0206293].

[7] R. Corcella et al., "HERWIG 6.5: an event generator for Hadron Emission Reactions With Interfering Gluons (including supersymmetric processes)”, JHEP 101 (2011) 010, [arXiv: hep-ph/0011363].

[8] S. Frixione and B. Webber, "Matching NLO QCD computations and parton shower simulations", JHEP 06 (2002) 029, [arXiv: hep-ph/ 020424 ] .

[9] S. Frixione, P. Nason, C. Oleari, Matching NLO QCD computations with Parton Shower simulations: the POWHEG method, JHEP 0711 (2007) 070, [arXiv: 0709.2092].

[10] S. Mrenna, T. Sjöstrand, P. Skands, "PYTHIA 6.4 physics and manual”, JHEP 05 (2006) 026, [arXiv: hep-ph/0603175].

[11] H.L. Lai et al., "New parton distributions for collider physics", Phys. Rev. D82 (2010) 074024, [arXiv:1007.2241].

[12] A.D. Martin et al., Parton distributions for the LHC, Eur. Phys. J. C63 (2009) 189, [arXiv:0901.0002].

[13] R. D. Ball et al., Parton distributions with LHC data, Nucl. Phys. B867 (2013) 244, [arXiv:1207.1303].

[14] H1 and ZEUS Collaboration, F.D. Aaron et al., "Combined Measurement and QCD analysis of the Inclusive ep Scat- tering Cross Sections at HERA”, JHEP 1001 (2010) 109, [arXiv: 0911.0884 ].

[15] ATLAS Collaboration, "Measurement of the differential cross-section of highly boosted top quarks as a function of their transverse momentum using the ATLAS detector in $\sqrt{s}=8 \mathrm{TeV}$ proton-proton collisions", ATLAS-CONF-2014-057 (2014) [https: / / cds . cern.ch/record/1951328]. 